\title{
EFEITOS DA RADIAÇÃO SOFT-LASER (DIODO) SOBRE O PROCESSO IFE CICATRIZAÇÃO CUTÂNEA EM FELINOS
}

THE IFFECT OF SOFT-LASER (IDIODES) RADIATION ON WOUND HEALING IN CATS

Júlia Maria MATF.RA'; Maria Lúcia Zaidan DA('Il'; Débora Bartalot PEREIRA'

\begin{abstract}
RESUNO
Veriticou-se o valor do emprego do laser diode (ja-As-SHI.D para anxiliar o processo de cicatrização cutânea em felinos. Utilizaram-se 21 animais que foram submetidos à ovariohisterectomia, 9 pertencendo ao grupo controle c 12 submetidos à laserterapia. (Os exames analomopatológicos foram realizados nos dias 2. 4 e 8 de pós-operatório. juntamente com avaliação macroscópica. Conclubu-se que a aplicação do laser diodo Ga-As-SHIL.D) aluxilion o processo cicatricial da ferida cirúrgica por aumento de resistência à tensão.
\end{abstract}

U NITERMIOS: I ausers; Diodo Gal-As-SHI.I); (icatrização: Felinos

\section{INTROIDLCÃO}

$O$ interesse pelos efejtos da luz monocromática coerente emitida pelo laser (light amplification by the stimulated emission of radiation) tem crescido muito nas duas últimas décadas. fenômeno este avaliado pela quantidade significativa de publicaçōes científicas, de experimentos controlados em animais de laboratório e relatos de casos clínicos.

Os chamados soft-lasers ou lasers de baixa energia, tais como laser de Hélio-Neônio. laser diodo, laser de rubi e laser de Argônio, estão sendo motivo de controvérsia no meio cienlífico: alguns pesquisadores alïmam que estes possuem efeito bioestimulante. outros contestam esta propriedade.

Dentro do capítulo da biocstimulação pelo soft-laser,o lópico que vem chamando a alenção dos cientistas é a aceleração da cicatrização das feridas de pele.

SILVER ${ }^{13}$ ( 1979 ) estuda o processo normal de cicatrização) cutânea e conclui que as estruturas epitcliais sofrem reparo através de regeneração, sem formação de cicalri\%; porém. os componentes do tecido conjuntivo podem ou não regenerar nos seus padrōes originais.

THOMISON ${ }^{16}$ (1984) descreve a cicatrização por primeira intenção da ferida cirúrgica, devido à proliferação de fibroblastos e células endoteliais, com produção de matriz e filamentos pelas mesmas no $2^{\prime \prime}$ ou $3^{\prime \prime}$ dia de pós-operatório, e colágeno escasso. Alguns meses foram necessários para que a resistência voltasse quase ao normal.

Atualmente, tenta-se provar a eficácia dos soft-lasers na cicatrizaçāo cutânea c estabelecer as dosagens para cada tipo de laser com seus respectivos comprimentos de onda, além das interferências que podem ocorrer devido a fatores inerentês à espécie. Fntretanto, diliculdades sāo encontradas para precisar os métodos de observação e mensuração da ação do laser sobre os tecidos cutâneos, que. basicamente, têm sido feitas com técnicas histológicas, medidas do tempo de fechamento da ferida, histometria c análises de imagens fotográficas da área da ferida.

HLTSCHENREITER et al. ' (1980) estudaram a ação da luz infravermelha e do laser de l leNe na cicatrização cutânea em ratos c observaram que não houve aceleração no processo cicatricial, mas um aumento da resistência à tensão nas feridas tratadas a laser.

KANA et al. ${ }^{8}$ (1981) investigaram o efeito do comprimento de onda do laser de HeNe e do laser de Argônio na cicatrização de feridas de pele em ratos. Concluíram que não houve diferença macroscópica na cicatrização do grupo controle e irradiado. A contração primária das feridas foi inibida com doses altas de irradiação laser, nos dois comprimentos de onda usados; com o laser de Argônio ocorreu aumento da

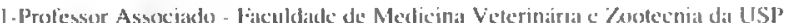

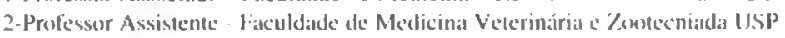

3-Médicas Vettrindria 


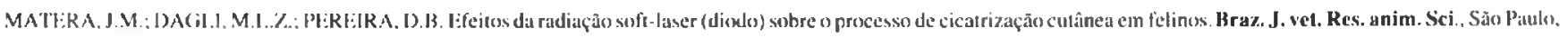
ง. 31. 11.1. . . 43-8. 1994.

síntese de colágeno e fïbroplasia; com o laser de HeNe a epitelização da ferida foi acelerada c houve aumento da produção de colágeno.

SURINCHAK et al. 15 (198.3) estudaram a contração de feridas cutâneas e a velocidadde de cicalrização $\mathrm{em}$ ratos e coelhos; usaram laser de HeNe, com diferentes tempos e doses de irradiação. Concluíram que não houve aceleração significativa na cicatrização, mas aumento na resistência à força de tensão.

JONGSMA et al. ${ }^{7}$ (198.3) investigaram a possibilidade de aceleração do lechamento de feridas cutâneas cm ratos, através de aplicação do laser de Argônio. Concluíram que não houve influência significante na cicatrização das feridas; sugerem que a estimulação do fechamento quando presente, é comprimento de onda dependente.

BOSATRA et al. ${ }^{2}$ (1984) estudaram a ação do laser de HeNe cm culturas de fibroblastos humanos da derme, e em úlceras de pacientes. Observaram, em ambos os casos, hipertrofia do retículo endoplasmático rugoso e do complexo de Golgi, com aumento da síntese de proteína por ação do laser, e desorganização da matriz intercelular colagenosa. Concluíram que há colagenogênese mais ativa dos fibroblastos, mas a síntese de fibrilas periódicas defïnitivas maturas é impedida por razões ainda desconhecidas.

VASCONCELOS et al. ${ }^{17}$ (1984) utilizaram o laser de baixa energia em casos clínicos com bons resultados na cicatrização cutânea.

COLLS CRUAÑES ${ }^{\dagger}$ ( 1984 ) conclui que a laserterapia tem efeitos bioestimulantes sobre a cicatrização cutânea, na dosagem de $4 J / \mathrm{cm}^{2}$, devido ao aumento na proliferação de células do tecido conjuntivo e estímulo para a neovascularização.

KOLARI" ( 1985 ) estuda as proriedades ópticas da pele para verificar a profundidade de penetração de luz na mesma, quando submetida à radiação do laser de HeNe e diodo GaAl-As. Concluiu que a transmitância da luz do laser diodo GaAl-As foi aproximadamente 1,6 vezes maior do que o laser de HeNe.

McCAUGHAN et al. " ( 1985 ) utilizaram laser de Argônio em cobaios, realizaram planimetria das áreas das feridas cutâneas para obter $\mathrm{em}$ dados quantitativos, mas não encontraram eleito estimulante na cicatrização.

SEKAN et al. ${ }^{12}$ (1986) citaram os seguintes efeitos do laser e suas aplicaçōes em cirurgia plástica: a) o efeito da radiação laser na pele depende da pigmentação e vascularização; b) a pele hiperqueratótica é resistente aos efeitos do laser; c) o uso de pigmentos artificiais escuros na superfície da pele tem efeito adjuvante; d) o efeito resultante depende do tipo de fonte de radiação laser, da duração da exposição, dos fatores ópticos e da quantidade de energia por $\mathrm{cm}^{2}$.

LONGO et al. ${ }^{10}$ (1987) realizaram experimento com ratos. comparando a cicatrização de leridas com uso do laser diodo Ga-As-Al, e a aplicação de fioestimulinas. Obtiveram resultados significantes de recpitelização e cicatrização das margens da ferida com a densidade de potência de $3 \mathrm{~J} / \mathrm{cm}^{2}$ durante 10 min., diariamente, durante 5 dias.

ANNEROTH et al.' (1988) trabalharam com laser infravermelho pulsátil de Ga-As-Al, em cicatrização de feridas; concluíram, que não há aceleração da cicatrização, tanto macro quanto microscopicamente, com até 14 dias de observação.

BRAVERMAN et al. ${ }^{3}$ (1989) irradiaram feridas de coelhos, com laser $\mathrm{HeNe}$, laser infravermelho pulsátil e a associação de ambos. Observaram resultados significativos, somente em relação à força de tensão.

HUBÁSEK e POSPÍSILOVÁ (1989) estudaram em ratos a influência do laser HeNe em feridas, através da atividade dos fibroblastos. Concluíram que nas áreas diretamente irradiadas, a laserterapia pode influenciar os períodos de cicatrização através de seu efeito na produção de tecido conjuntivo.

O presente trabalho tem como objetivo avaliar o processo de cicatrização de feridas cirúrgicas assépticas em felinos submetidos à irradiação laser do diodo Ga-As-SHLD, através de métodos qualitativos de observação clínica e exame histopatológico.

\section{MATERIAI, F MÉTODO}

Utilizamos 21 fêmeas da espécie felina, adultas, com diferentes idades e raças, que foram encaminhadas ao Setor de Técnica Cirúrgica do Hospital Veterinário da Faculdade de Medicina Veterinária e Zootecnia da Universidade de Sắo Paulo, para serem submetidos à ovariohisterectomia.

Os 21 animais foram divididos em 6 grupos, e distribuidos da seguinte maneira:

Grupo 1: exposto ao laser no dia da cirurgia. Biópsia e observações clínicas macroscópicas no $2^{\circ}$ dia de pós-operatório;

Grupo 2: controle. Biópsia e observações clínicas macroscópicas no $2^{\circ}$ dia de pós-operatório; 


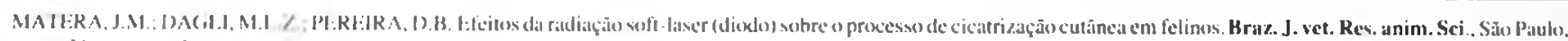

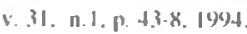

Grupo 3: exposto ao laser no dia da cirurgia. Biópsia e observações clínicas macroscópicas no 4" dia de pós-operatório;

Grupo 4: controle. Biópsia e observaçóes clínicas macroscópicas no 4" dia de pós-operatório:

Grupo 5: exposto ao laser no dia da cirurgia. Biópsia e observações clínicas macroscópicas no 8 " dial de pós-operalório:

Grupo 6: controle. Biópsia e observaçoes clinicas macroscópicas no 8" dial de pós-operatório.

Os grupos lestes e controles foram constituídos por 4 a 3 fêmeas cadat. respectivamente.

Após os cuidados pré-operatórios usuais. estas fêmeas receheram como pré-inestesia sulfato de atropina $0.25 \mathrm{mg}$ na dose de $0 .(04+\mathrm{mg} / \mathrm{kg}$ e como anestesia uma mistura de cloridrato de tiletamina e cloridrato de zolazepam*, na dose de 0.15 a $0.2 \mathrm{ml} / \mathrm{kg}$, via subcutânca, complementada por inalação com halotano** e oxigênio, por meio de aparelho de anestesia em circuito aberto.

Todas as fêmeas foram submetidas à ovariohisterectomia pela técnica apresentada por ST( )NE: ${ }^{4}$ ( 1985$)$; 0 comprimento da incisão cirúrgica foi sempre de $6 \mathrm{~cm}$. e a sutura da pele com fio de algodão*** em pontos simples separados.

Após o término da cirurgia. os animais pertencentes aos grupos 1,3 e 5 foram irradiados com um emissor de laser diodo Ga-As-SIIL,J), pulsátil, comprimento de onda de 9()4 nm. densidade de energia de $2 \mathrm{~J} / \mathrm{cm}^{2}$ e tempo de exposição automaticamentc ajustide pelo aparelho****. () depósito de encrgia era feito no intervalo entre cada ponto simples separado, com a cabeça do laser perpendicular à pele.

A seguir, uma bandagem de gaze estéril e esparadrapo cra colocada sobre a sutura.

Para a realização das biópsias os animais receberam os mesmos cuidados pré-operatórios e foram submetidos ao mesmo tipo de pré-anestesia e anestesia. (Os fragmentos de pele c tecido subcutâneo foram coletados incluindo um ponto simples separado de algodĩo da sutura original, formando um yuadrado de aproximadamente $1 \mathrm{~cm}^{2}$ de área. (Os fragmentos foram fixados em formalina a $10 \%$, e processados pelas técnicas usuais de inclusão em paralina. e os cortes de 5 micrômetros obtidos foram corados pelo método de hematoxilina-cosina. As lâminas assim obtidas foram exami-

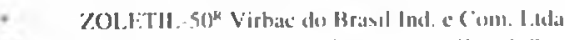

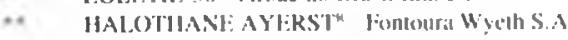

nadas em microscópio óptico comum.

() protocolo utilizado para o exame macroscópico no dia da biópsia foi o seguinte:

N"de Identificação: Raça: Sexo: Idade: Pelagem:

Nome: Data da Cirurgia: Data da biópsia:

Laser: aplicação de $2 \mathrm{~J} / \mathrm{cm}^{2}$.

\section{EFEITOS GERAIS}

Temperatura corpórea: Mucosas: Palpação abdominal:

Outras observações:

\section{FERII)A CIRÚRGICA}

Temperatura local: Normal: Aumentada: Diminuida:

Presença de secreçāo: Sim: Não:

Tipo: Quantidade:

Coloração da cicatriz: Rósea: Vermelha:

Com vascularização: Sim: Não:

Na cicatriz (linha de incisão):

Ao redor do fio (entrada/saída):

Resistência tênsil do fio: Normal: Aumentada: Diminuída:

Resistência da ferida cirúrgica:

1 : Deiscência:

II : Separação espontânea (tendência à deiscência):

III : Separação dos bordos da ferida sob leve tensão:

IV : Cicatrização sem possibilidade de separação do bordo da ferida sob leve tensão:

Retração da ferida:

Outras observaçōes:

Os exames da temperatura local, resistência tênsil do fïo e da ferida cirúrgica foram realizados manualmente.

** HTHICON" - Johnson \& Johnoon.

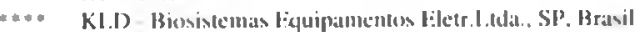




\section{RESUITAIOS}

\section{A) Observações clínicas macroscópicas}

()s resultados obtidos nos grupos controles e irradiados com laser encontram-se resumidos nas Tab. I e 2.

\section{B) Observaçōes anatomopalológicas microscópicas}

O exame histopatológico dos cortes de peles irradiadas revelou seqüencia de allerações características de um processo normal de cicatrização por primeira intenção.

No 2" dia de pós-operatório observou-se o início do processo de inversão da epiderme. Na derme profunda e hipoderme havia processo inflamatório agudo constituído principalmente por polimorfonucleares distribuídos difusamente e ao redor dos vasos sanguíneos (perivasculite). Foi observado ainda o início de um afluxo de fibroblastos, sendo que estes apresentavam-se alongados, com núcleos ovalados. de tamanho grande e nucléolos evidentes. Na derme o colágeno mostravase denso, hialinizado e fragmentado. Ao redor do lio de sutura havia processo inflamatório agudo, com grande quantidade de fibrina.

No 4" dia de pós-operatório caracterizou-se a inversào epidérnica com hiperplasia desta camada. Basicamente as mesmas alteraçôes do 2" dia de pós-operatório foram constaladas na derme. além da observação do início da neovascularização. Tais vasos mostravam-se de contornos irregulares e delimitados por células endoteliais com núcleo grande ovalado ou arredondado.

No 8" dia pós-operatório a inversãoepidérmica com hiperplasia persistiu. Alteraçôes referentes alo colágeno dérmico não foram observadas. Va derme profunda e hipoderme constatou-se a presença de tecido de granulação, com proliferação de fibroblastos, vasos neoformados e colágeno. Ao redor do fïo de sutura observou-se processo inllamatório crônico com mononucleares e componentes de lecido de granulação, acrescido de células gigantes do tipo corpo estranho.

Não foram constatadas alteraçoes histopatológicas significantemente diferentes nos animais controle.

\section{DISCUSSก்̃}

Observamos nox pacientes dos grupos irradiados um aumento da resistência da borda da ferida quando submetidos à tensão a partir do 2" dia de pós-operatório, sendo mais significativo a partir do 4"dia de pós-operatório, comparando-se ao grupo controle, confirmando os resultados de HUTSCHENREITER et al. ${ }^{\circ}(1980) ;$ SURINCHAK et al. ${ }^{15}$ (1983); ANNEROTH et al. ${ }^{1}$ (1988) e BRAVERMAN el al. ${ }^{3}$ (1989).

Constatamos que a cicatrização macroscópica do $8^{\circ}$ dia de evolução pós-operatória, quando se dá a retirada convencional dos pontos, mostrou-se sem diferença significativa entre os grupos controles e irradiados, havendo cicatrização por primeira intençāo em ambos. Este resultado vem ao encontro das citações de literatura, HUTSCHENREITER el al. ${ }^{6}(1980)$; KA.NA et al. ${ }^{x}$ (1981); JONGSMA et al. ' (198.3); SURINCHAK et al. " (1983); McCALGIIAN el al." (1985); ANNEROTH et al." (1988) e BRAVERMAN et al. "(1989), apesar de utilizarem diferentes tempos de exposição e dose.

No tocante aos resultados histopatológicos, observamos um afluxo de fibroblastos e infiltrado inflamatório a partir do $2^{\circ}$ dia de pós-operatório; início de neovascularização ao $4^{\circ}$ dia de pós-operatório, mencionado por COOLS CRUAÑES (1984); HUBÁCEK: POSPÍSIL OVÁs (1989). Ao contrário do que verificamos, LONGO et al. ${ }^{10}$ (1987) relataram ausência de infiltrado inflamatório na derme, embora o tempo de exposição e a dosc utilizada tenham sido maiores.

O método de coloração pela hematoxilina-eosina por nós empregado não permitiu constatar diferenças quanto ao tipo de colágeno em ambos os grupos, porém, com a técnica de coloração Picrosirius red ou com imunohistoquímica, talvę isso losse viável.

A maioria dos trabalhos reportados utilizaram espécies diferentes da nossa, podendo trazer algumas variações nos resultados obtidos.

\section{CONCIUSÖES}

Segundo a metodologia empregada para a avaliação póscirúrgica em felinos submetidos à ovariohisterectomia e aplicação de laserterapia, julgamos poder concluir que:

1) a aplicação de laser diodo Ga-As-SHLD em felinos auxilia o processo cicatricial da lerida cirúrgica por. aumento da resistência à tensão;

2) o aparelho de laser diodo Ga-As-SHLD é de fácil aplicação. sendo necessário conhecimento das doses preconizadas e seus efeitos colaterais:

3) a utilização do laser diodo (ia-As-SHLD na espécie felina poderá ser de grande valia, se novos ensaios vierem a ser realizados, utilizando-se novas técnicas anatomopatológicas para sua avaliação. 


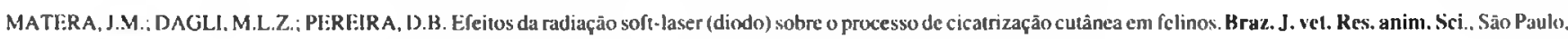
v. 31. n.1. p. 43-8, 1994

TABELA 1

Efeito da radiaçāo "soft-laser" (diodo) Ga-As-SHI.D sobre feridas cirúrgicas cutâneas (observações macroscópicas) em felinos fêmeass, SRD. Grupos controles. São Paulo, 1991.

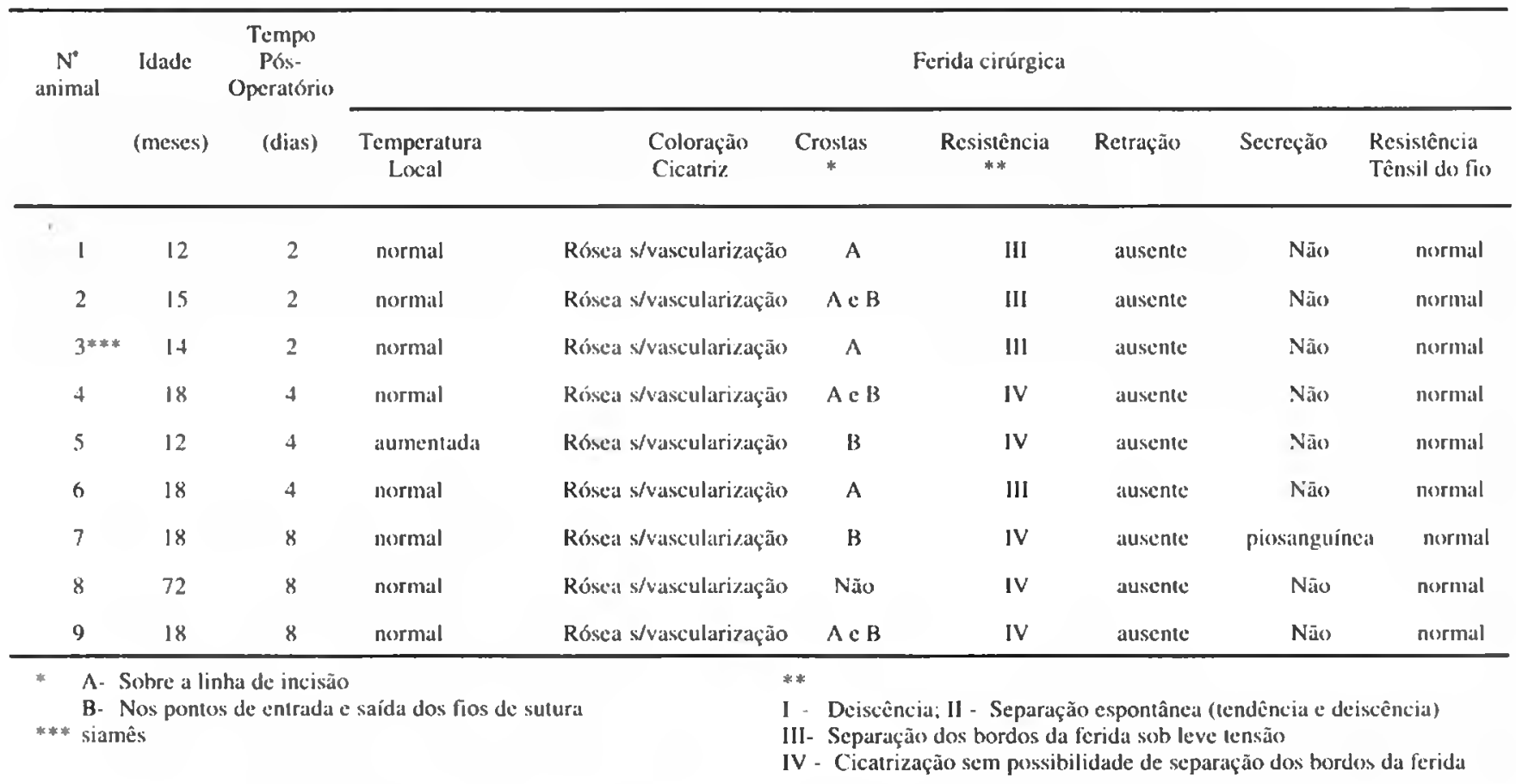

TABELA 2

Efeito da radiação "soft-laıser" (diodo) Ga-As-SHILD (2J/cm²) sobre feridas cirúrgicas cutâneas (observações macroscópicas) em felinos fêmeas, SRD. Grupos testes. São Paulo, 1991.

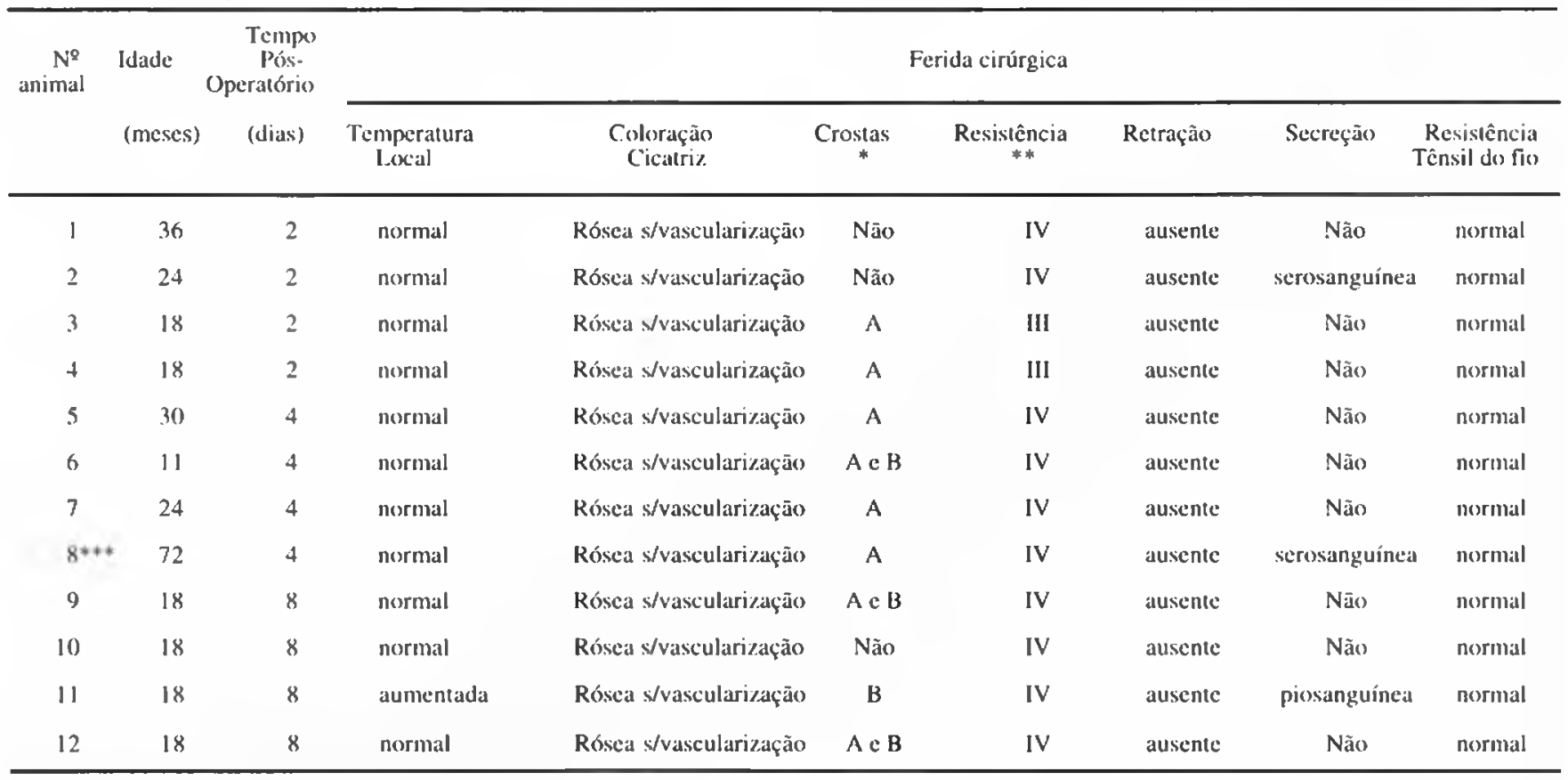

* A- Sobre a linha de incisão

B- Nos pontos de entrada e saída dos fíos de sutura *** siamês
I - Deiscência; Il - Separaçāo espontânea (tendência c deiscência)

III - Separação dos bordos da ferida sob leve tensão

IV - Cicatrização sem possibilidade de separação dos bordos da ferida 


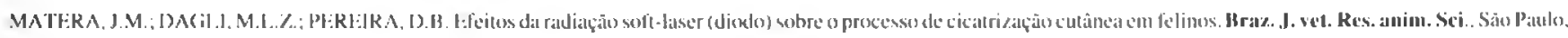
v.31, n.1. p. 4.3-8, 109.

\section{SLMMARY}

The main objective of this investigation was to evaluate the improvement in skin wound healing in cats with the use of soft laser (diodes) Ga-As-SHLD radiation (9)( $14 \mathrm{~nm})$. Ovariohisterectomised cats were divided into a control group and a laser-treated group. Anatomopathological observations were taken on days 2, 4 and 8 , post-surgery along with macroscopic evaluation. We concluded that laser diode Ga-As-SHI.D application ameliorated the surgical wound healing process by increasing its tensile strength.

UNITERMS: Lasers; Diodes (ia-As-SHID); Healing: Calls

\section{RHFERÊNCIAS BIBI.IOGRÁFICAS}

()!-ANNEROTH, G.; HALL, G.; RYDÉN, H.: ZLETTERQVIST, L. The effect of low-energy infrared laser radiation on wound healing in rats. Brit.J.Oral Maxillof.Surg., v. 26, p. 12-7, 1988.

()-BOSATRA, M.: JUCCI, A.; OLLIARO, P.: QUACCI. I).; SACCHI, S. In vitro fibroblast and dermis fibroblast activation by laser irradiation at low encrgy. Dematologica, v. 168. p.157-62, 1984.

03-BRAVERMAN, B.: MCCARTHY, R.J.; IVANKOVICH. A.I.: FORIDE, D.E.; (OVERFIELI), M.: BAPNA., M.S. Feffect of helium-ncon and infrared laser irradiation on wound healing in rabbits. Lasers Surg.Med.. v.9, p.50-8. 1989.

04-COLLS CRUAÑES, J. I a terapia laser, hoy. Barcelona. Centro Documentacion Láser de Meditec. S.A., 1984. p. 1]-3, 39-43, 52-9, 138-40.

05-HLBÁCEK, J.; POSPÍSII OVÁ, J. Becinllussung der wundregeneration durch lasereinwirkung auf libroblasten. Acta Universitatis Palackianac Olomucensis Facultatis Medicae. v.122. p.3II-6, 1989.

(6)-HUTSCHENREITER, G.: HAINA, 1).: PAULINI. K.; SCHLMACHER, G. Wundheilung nach laser und rollichthestrahlung.Z.Exp.Chir., v. 13, p.75-85. 1980.

()7-JONGSMA, F.H.M.; BOGAARD. A.E.J.M.; Van (iERMERT, M.J.C; HENNING, J.P.H. Is closure of open skin wound in rats accelerated by argon laser exposure? I asers Surg.Med., v..3, p. 75-80) 1983.

08-KANA, J.S.; HUTSCHENREITER, G.: HAINA, D.: WAIDELICH, W. Effect of low-power density laser radiation on healing of open skin wound in rats. Arch.Surg., y.116, p.293-6, 1981.
(09-KOLARI, P.J. Penetration of unfocused laser light into the skin. Arch.Dermatol.Res., v.277, p..342-4, 1985.

10-LONGO, L.; EVANGEI ISTA, S.; TINACCI. (i.; SESTI, A.G. Effect of diodes-laser silver-arsenide-aluminium (Ga-Al-As) 904 nm on healing of experimental wounds. Lasers Surg.Med., v.7, p.444-7, 1987.

11-MCCAUGHA.V J.S.; BETHEL, B.H.; JOHNSTON, T.: JANSSEN, W. Effect ol low-dose argon irradiation on rate of wound closure. I asers Surg.Med., v.5, p. $607-$ 14, 1985.

12-SIKKAN, V.; BROZMAN, M.: ZBOJA. S.; JANOVIC, J. Effect of laser rays on the skin and laser for plastic surgery uses. Acta Chirur. Plast., v.28, p. I-6, 1986.

13-SII.VI:R, I.A. The mechanies of wound healing. Fquine Vet. J.. v.11, p.93-6. 1979.

14-STONF, E.A.: In: SLATTER. D.H. Textbook of small animal surgery. Philadelphia, W.B. Saunders, 1985. p. $1660-1$.

15-SURINCHAK, J.S.; ALAGO, M. 1.,; BEI.LAMY, R.F.; STUCK, B.E.; BELKIN, M. Effects of low-level energy lasers on the healing of full-thickness skin defects. I asers Surg. Med.. v.2, p.267-74, 1983.

16-TH()MPSON, R.(i. Anatomia patológica general veterinária. Zaragoza, Acribia, 1984. p. 269-91.

17-VASCONCELOS, (j.F.; IERREIRA, A.J.A.: OLIVEI. RA, A.S.L. () laserer mole e sua aplicação clínica em medicina veterinária (nota prévia). Rev. Port. Ciênc. Vet., v.79, n.472, p.291-8. 1984.

Recebido para publicação em 27/07/92 Aprovado para publicação em 10/03/93 